\title{
Supply Chain Coordination Based on Buyback Contract under the Imperfect Product Quality and Fuzzy Demand Environments
}

\author{
Xiaobin Wang, Yihua Wang \\ School of Management Science and Engineering, Shandong University of Finance and Economics, Ji'nan 250014, China \\ wangxb@sdufe.edu.cn, wyhwyp1@126.com
}

\begin{abstract}
The coordination of two-echelons supply chain by buyback contract under the imperfect product quality and fuzzy demand environment is investigated in this paper. The mathematical models are built based on credibility theory, and the optimal parameters of buyback contract are obtained. In addition, the influence of the imperfect product rate on the parameters of buyback contract is analyzed. Finally, a numerical example is presented to demonstrate the solving processes of the models and the different effects of imperfect product rate on parameters of buyback contract and the fuzzy expected profit value of all members in supply chain.
\end{abstract}

Keywords- supply chain; buyback contract; fuzzy demand; credibility theory

\section{INTRODUCTION}

In recent years, the practices and research of supply chain management widely emerged around the world, and there are many useful results arisen from the theoretical and practical aspects. For the successful management of supply chain, it should be maximizing both the profits of all participants and the profits of the entire supply chain. Therefore how to establish one mechanism for coordinating the benefit in supply chain has become one of the key issues in supply chain management. So far, there are a lot of researches and practical explorations found that the contract is an effective mechanism to achieve coordination in supply chain. Generally, supply chain coordination contract includes of wholesale price contract, buyback contract, revenue-sharing contract, quantity-flexibility contract and others ${ }^{[1]}$. The socalled buyback contract, it refers to the supplier sell the unit items with a wholesale price to the retailer, but pays the retailer a buyback price per unit remaining at the end of the season. Under the random market demand, many studies have proved that buyback contract is effective for the realization of supply chain coordination. For example, Emmons and Gilbert ${ }^{[2]}$, Padmanabhan and Png ${ }^{[3]}$, Wang ${ }^{[4]}$, Webster $^{[5]}$ and $\mathrm{Yao}^{[6]}$ have investigated the problem from a different perspective study on the effectiveness of the buyback contracts on supply chain coordination. From the researches mentioned above, it can be found that the uncertainties of the market demand in most cases are described as random phenomenon, that is, demand is a random variable. However if the demand be taken as a random variable, which should be known its probability distribution, but it is difficult or impossible in reality to obtain the distribution information to the market demand due to the rapidly changing market conditions. In most cases, the estimation of the demand is depending on the experiences and judgment of senior management. From the point of this view, the demand is suitable to regard as fuzzy variable rather than random variable, and the approach of fuzzy information processing should be used for doing some researches. In recent years, with the development of fuzzy theory, many scholars have begun to focus on research of supply chain coordination under uncertainty environment based on fuzzy theory. So far, there are few researches about supply chain coordination with buyback contract under fuzzy demand environment. Zhao et al. ${ }^{[7]}$ treated the market demand as random variable firstly, and then considered the fuzzy random demand updating; they explored the response method for buyback contract in supply chain by applying fuzzy set theory and probability theory. But in those study as well as most other studies, one of the fundamental assumptions is that the products supplied by suppliers are defect free. However in reality, due to lack of control over the production process, impact of natural disasters, transport damage factors in the process, there are some defective products often being found in the orders or deliveries, and this session has investigated in supply chain coordination problem with the consideration of the random demand by many scholars. But under the fuzzy demand environment, the study was rare. In 2010 , Liu and Chen ${ }^{[8]}$ incorporated the product defect rate into the buyback contract, and treated the product defect rate and coordination parameters as fuzzy numbers, and they studied how the defect rate impact the parameters in buyback contract based on function principle and graded mean integration representation. But in the literature [7][8], there are still some problems on the method be used to build the model. Therefore, with the consideration of product defect rate and fuzzy demand situation, it is necessary to explore new theories and methods of supply chain coordination with buyback contract validity. Credibility theory was proposed in 2004 by Chinese scholars Liu Baoding ${ }^{[9]}$ and which has formed a complete axiomatic system and also is a new branch of the fuzzy theory. In recent years, credibility theory is also applied by some scholars to researches of supply chain optimization problems. For example, Wang and wang ${ }^{[10]}$ studied buyback contract in a supply chain with fuzzy demand, and presented the optimal order quantity for total supply chain and analyzed how to affect the contract for the variety of cost parameters in supply chain circumstances. Sang et al. ${ }^{[1]}$ considered the supply chain coordination based on revenue share contract and buyback contract under fuzzy demand environment, but they did not consider the impact of defective products for contract parameters. This article summarizes the research results till now and investigates the supply chain 
coordination problem with buyback contract under production defects and fuzzy demand environment based on credibility theory. And also we assume that the defective product has a certain residual value, then by constructing the fuzzy models and solving them, the relationship between the parameters of buyback contract and defect rate is discussed and the impact of defect rate on the variety of parameters are presented by a numerical example.

\section{PRELIMINARIES}

Credibility measure was used to measure the fuzzy event and fuzzy phenomenon, and which is a self-dual measure. Let $\Theta$ be a nonempty set, $P(\Theta)$ the power set of $\Theta$, for an element $\mathrm{A} \in P(\Theta), \operatorname{Cr}\{\mathrm{A}\}$ expresses the chance that fuzzy event A occurs and is called a credibility measure if and only if

(1) $\operatorname{Cr}(\Theta)=1$.

(2) $\operatorname{Cr}\{\mathrm{A}\} \leq \mathrm{Cr}\{\mathrm{B}\}$ whenever $\mathrm{A} \subset \mathrm{B}$.

(3) $\mathrm{Cr}$ is self-dual, that is, $\operatorname{Cr}\{\mathrm{A}\}+\operatorname{Cr}\left\{\mathrm{A}^{c}\right\}=1$ for any $A \in P(\Theta)$.

(4) $\operatorname{Cr}\left\{\bigcup_{i} \mathrm{~A}_{i}\right\} \wedge 0.5=\sup _{i} \operatorname{Cr}\left\{\mathrm{A}_{i}\right\}$ for any $\left\{\mathrm{A}_{i}\right\}$ with $\operatorname{Cr}\left\{\mathrm{A}_{i}\right\} \leq 0.5$.

Then the triplet $(\Theta, P(\Theta), \mathrm{Cr})$ is called a credibility space, and a fuzzy variable is defined as a function from the credibility space $(\Theta, P(\Theta), \mathrm{Cr})$ to the set of real numbers. (see [9])

Definition 1: Let $\xi$ be a fuzzy variable on a credibility space $(\Theta, P(\Theta), \mathrm{Cr})$. Then the expected value $E[\xi]$ is defined as

$$
E[\xi]=\int_{0}^{+\infty} \operatorname{Cr}\{\xi \geq r\} \mathrm{d} r-\int_{-\infty}^{0} \operatorname{Cr}\{\xi \leq r\} \mathrm{d} r
$$

provided that at least one of the two integrals is finite.

Definition $2^{[9]}$ : Let $\xi$ be a fuzzy variable on a credibility space $(\Theta, P(\Theta), \mathrm{Cr})$. If the credibility distribution $\Phi[-\infty,+\infty] \rightarrow[0,1]$ satisfied $\Phi(x)=\operatorname{Cr}\{\theta \in \Theta \mid \xi(\theta) \leq x\}$, then $\Phi$ is named the credibility distribution of $\xi$.

Definition $3^{[9]}$ : Let $\xi$ be a fuzzy variable on a credibility space $(\Theta, P(\Theta), \mathrm{Cr})$, Then the credibility density function $\varphi: \mathrm{R} \rightarrow[0,+\infty)$ of the fuzzy variable $\xi$ is a function such that $\Phi(x)=\int_{-\infty}^{x} \varphi(y) \mathrm{d} y, \forall x \in \Re$, and $\int_{-\infty}^{+\infty} \varphi(y) \mathrm{d} y=1$, where $\Phi$ is the credibility distribution of the fuzzy variable $\xi$.

Lemma $1^{[9]}$ : Let $f$ be a function on $\mathrm{R} \rightarrow \mathrm{R}$ and $\xi$ a fuzzy variable defined on a credibility space $(\Theta, P(\Theta), \mathrm{Cr})$. Then the expected value $E[f(\xi)]$ is defined as

$$
E[f(\xi)]=\int_{0}^{+\infty} \operatorname{Cr}\{f(\xi) \geq r\} \mathrm{d} r-\int_{-\infty}^{0} \operatorname{Cr}\{f(\xi) \leq r\} \mathrm{d} r,
$$

provided that at least one of the two integrals is finite.

Lemma $2^{[12]}$ : Let $\xi$ be a fuzzy variable with distribution function $\Phi(\cdot)$ and credibility density function $\varphi(\cdot)$. Assume that the credibility distribution's support is $\Theta=[u, v]$. Then

$$
E[\min (z, \xi)]=z-\int_{u}^{z}(z-x) \varphi(x) \mathrm{d} x \text {, where } 0 \leq u \leq z \leq v
$$

and

$$
\frac{\mathrm{d} E[\min (z, \xi)]}{\mathrm{d} z}=1-\Phi(z) .
$$

\section{PROBLEM DESCRIPTION AND FORMULATION}

Considering a two-echelon supply chain includes one supplier and one retailer, and in which the supplier products and supplies new products to the retailer, due to lack of historical data about demand or some dates are derived from the expert's estimate or judgment. So the demand in the market should be characterized as a fuzzy variable. In addition, when the new products are brought to the market, it's hard to avoid the occurrence of the defect product. Here, we assume that the defect product has a certain residual value, but its testing cost is small and can be negligible. For the recycling of the defect product, it is full responsibility for the supplier. Retailers make the order to the vendor before the sales, and if the demand is less than the retailer's order, retailers can return the unsold products to the supplier with a pre-decided buyback price at the end of the period, also it is assumed that the buyback price of the defect product are similar with the buyback price of the normal product. Parameters mainly used in the study are as follows: $C_{\mathrm{S}}$ unit product cost for supplier; $C_{\mathrm{R}}$ unit dealing cost for retailer; $p$ price of the product; $w$ Wholesale prices per product suppliers to retailers (decision variable); $q$ order quantity of the retailer; $b$ buy back price $(b<w<p)$; $D$ market demand; $\alpha$ defect rate; $v(p)$ residual value of unit product, $v(p)=\lambda p<b,(0<\lambda<1) ; \pi_{R}, \pi_{S}, \pi_{S C}$ express the profit of retailer, supplier and the whole supply chain respectively. If the defect rate of the product is 0 , that means the defect product is not exist. Then the profit of the retailer is composed of the income from sales, returns and also gets rid of the purchase cost and the processing cost, and which can be expressed as:

$$
\pi_{R}=p \min (q, D)+b \max (q-D, 0)-\left(w+C_{R}\right) q .
$$

The profit of the supplier is composed of the income from wholesales, residual value of the returns and also gets rid of the production cost and the buyback cost, and which can be expressed as:

$$
\pi_{S}=\left(w-C_{S}\right) q+v(p) \max (q-D, 0)-b \max (q-D, 0) .
$$

The profit of supply chain is: 


$$
\pi_{S C}=p \min (q, D)+v(p) \max (q-D, 0)-\left(C_{R}+C_{S}\right) q .
$$

For the whole supply chain system, the decision is to find the optimal order quantity $q^{*}$, and which should be the optimal solution of the following programming problem

$$
\begin{aligned}
& \max E\left[\pi_{S C}\right] \\
& \text { s.t. } q>0
\end{aligned}
$$

The Programming problem (5) is a typical nonlinear programming problem, and applying the solving method of the nonlinear programming, its optimal solution should be:

$$
q=\Phi^{-1}\left(\frac{p-C_{R}-C_{S}}{(1-\lambda) p}\right)
$$

If the defect rate is not zero, that is, when there are some defective products, according to the previous assumption that after the recovery of the residual value per product $v(p)=\lambda p,(0<\lambda<1)$; In addition, since the defect rate is $\alpha$, so the perfect product in each order is $q^{\prime}=(1-\alpha) q$, In the fuzzy demand environment, the retailer, supplier and the whole supply chain system's profit expressed as:

$$
\begin{aligned}
\pi_{R}^{\prime} & =p \min \left(q^{\prime}, \mathrm{D}\right)+b \max \left(q^{\prime}-\mathrm{D}, 0\right)+b \alpha q-C_{R} q-w q \\
& =(p-b) \min \{(1-\alpha) q, \mathrm{D}\}+b q-C_{R} q-w q, \\
\pi_{S}^{\prime} & =\left(w-C_{S}\right) q-b \max \left(q^{\prime}-D, 0\right)+v(p) \max \left(q^{\prime}-D, 0\right)-b \alpha q+v(p) \alpha q \\
& =(b-\lambda p) \min \{(1-\alpha) q, D\}+\left(w-C_{S}+\lambda p-b\right) q,
\end{aligned}
$$

and

$$
\begin{aligned}
\pi_{S C}^{\prime} & =p \min \left(q^{\prime}, D\right)+v(p) \max \left(q^{\prime}-D, 0\right)-C_{R} q-C_{S} q+v(p) \alpha q \\
& =p(1-\lambda) \min \{(1-\alpha) q, D\}+\lambda p q-\left(C_{R}+C_{S}\right) q .
\end{aligned}
$$

Since the demand $D$ is a fuzzy variable, and its credibility distribution is $\Phi(\cdot)$, credibility density function $\varphi(\cdot)$, following from the (3), (4) in Lemma 2 that we have

$$
\begin{aligned}
E\left[\pi_{R}^{\prime}\right]= & E\left[(p-b) \min \{(1-\alpha) q, D\}+b q-C_{R} q-w q\right] \\
& =(p-b) E[\min \{(1-\alpha) q, D\}]+\left(b-C_{R}-w\right) q .
\end{aligned}
$$

In order to obtain the optimal order quantity, with the similar method to solve the programming problem; Take the first and second derivatives of $E\left[\pi_{R}^{\prime}\right]$ with respect to $q$, then we have

$$
\frac{\mathrm{d} E\left[\pi_{R}^{\prime}\right]}{\mathrm{d} q}=(1-\alpha)(p-b)(1-\Phi((1-\alpha) q))+b-C_{R}-w
$$

and

$$
\frac{\mathrm{d}^{2} E\left[\pi_{R}^{\prime}\right]}{\mathrm{d} q^{2}}=(1-\alpha)^{2}(b-p) \varphi((1-\alpha) q) \leq 0 .
$$

Let $(7)=0$ and then we can obtain the optimal order quantity of the retailer is

$$
q^{*}=\frac{\Phi^{-1}\left(\frac{(1-\alpha) p+\alpha b-w-C_{R}}{(1-\alpha)(p-b)}\right)}{1-\alpha} .
$$

And also the expected total profit of supply chain is

$E\left[\pi_{s C}^{\prime}\right]=p(1-\lambda) E[\min \{(1-\alpha) q, D\}]+\lambda p q-\left(C_{R}+C_{S}\right) q$.

Similarly, we can obtain that the optimal order quantity for the supply chain is

$$
q^{* *}=\frac{\Phi^{-1}\left(\frac{p-\alpha p+\alpha \lambda p-C_{R}-C_{S}}{(1-\lambda)(1-\alpha) p}\right)}{1-\alpha} .
$$

From (9), we know that when $\alpha=0$, the value of $q^{* *}$ is equal to the value of $q$ in (6).

Since the expected profit of the supplier is $E\left[\pi_{S}^{\prime}\right]=(b-\lambda p) E[\min \{(1-\alpha) q, D\}]+\left(w-C_{S}+\lambda p-b\right) q$, similarly, we can obtain the optimal supply quantity of the supplier as,

$$
q^{* * *}=\frac{\Phi^{-1}\left(\frac{w-C_{S}+\alpha \lambda p-\alpha b}{(1-\alpha)(b-\lambda p)}\right)}{1-\alpha} .
$$

If buyback contract can coordinate the supply chain, which should satisfy $q^{*}=q^{* *}=q^{* * *}$, from that we have

$$
w=\frac{p-C_{R}-C_{S}}{(1-\lambda) p} b+\frac{C_{S}+\lambda C_{R}-\lambda p}{1-\lambda} .
$$

From (10) we know that when $p, C_{R}, C_{S}$ and $\lambda$ are determinate, there has a line relationship between $w$ and $b$. By the way, the equation (10) is independent to $\alpha$. From the point of this view, we introduce a parameter $\eta(0<\eta<1)$, and also suppose that $w$ and $b$ are the function of $\eta$, then $w$ and $b$ can be expressed as:

$$
\left\{\begin{array}{l}
w(\eta)=\eta\left(p-C_{R}-C_{S}\right)+C_{S} \\
b(\eta)=\eta(1-\lambda) p+\lambda p .
\end{array}\right.
$$

In fact, from (11) and $w>C_{S}$ we know that if $p-C_{R}-C_{S}>0$ then $\eta>0$. Since $p>C_{R}+C_{S}$ and $b<w$ is obvious in real supply chain, instituting the value in (11) into it and we can obtain the value of parameter $\eta$ satisfied the expression as follows.

$$
\left(C_{R}+C_{S}-\lambda p\right) \eta<C_{S}-\lambda p,
$$

If $C_{S}>\lambda p$, then the parameter $\eta$ satisfied

$$
0<\eta<\frac{C_{S}-\lambda p}{C_{R}+C_{S}-\lambda p}<1
$$

Hence, the parameter $\eta$ introduced above satisfied the assumptions $0<\eta<1$ if $C_{R}+C_{S}<p$ and $\lambda p<C_{S}$. From (11) we have $w-b=\eta\left(\lambda p-C_{R}-C_{S}\right)+C_{S}-\lambda p$, so when $p, C_{R}, C_{S}$ and $\lambda$ are fixed, if $\eta$ increase, then $w-b$ tend to decrease. This means, with the bargaining power of supplier enhancing, it is benefit for supplier to provide the buyback price being close to the wholesale. But in order to enable the retailer to accept buyback contract for achieving supply chain coordination, we should have $b<\frac{p\left(C_{S}+\lambda C_{R}-\lambda p\right)}{C_{R}+C_{S}-\lambda p}$ 
with the conditions that (10) and $w>b$ holds. From (10) and (11), we know that the values of $w$ and $b$ are independent to $\alpha$, but from (8) and (9), we know that the values of $q^{*}, q^{* *}$ and $q^{* * *}$ are dependent on the vary of $\alpha$. However, the trends of the change about values of $q^{*}$, $q^{* *}$ and $q^{* * *}$ do not have clear analysis result. Only if $q^{*}=q^{* *}=q^{* * *}$, then the supply chain can be coordinated. In the next section, a numerical example will be presented to analyze the impact of $\alpha$ on the varieties of $q^{*}$, $q * *$ and $q * * *$.

\section{NUMERICAL EXAMPLES}

In order to illustrate the effectiveness of the models in coordinating the supply chain, a numerical example is presented in the following. Suppose that the demand of market $D$ is a triangle fuzzy variable, and $D=(1000,1100$, 1200), $\eta$ is 0.6 , and $\alpha$ take values of $0,0.02,0.03$ and 0.045 in different cases, respectively, other parameters and their values are $p=40, C_{S}=10, C_{\mathrm{R}}=2$ and $\lambda=0.1$, calculation result is listed in Table 1 when applying the models to deal with this problem.

TABLE 1: THE RESULT OF COORDINATION WITH GIVEN $(\eta, \alpha)$

\begin{tabular}{|lcccl|}
\hline \multicolumn{1}{|c}{$(\eta, \alpha)$} & $b$ & $w$ & $q^{*}$ & $E\left[\pi_{R}^{\prime}\right]$ \\
\hline$(0.60,0)$ & 25.6 & 26.8 & 1155.6 & 12071.1 \\
$(0.60,0.02)$ & 25.6 & 26.8 & 1178.2 & 11995.7 \\
$(0.60,0.03)$ & 25.6 & 26.8 & 1189.9 & 11956.8 \\
$(0.60,0.045)$ & 25.6 & 26.8 & 1207.8 & 11897 \\
$(0.60,0.05)$ & 25.6 & 26.8 & 1213.9 & 11876.7 \\
$(0.60,0.06)$ & 25.6 & 26.8 & 1226.3 & 11835.4 \\
$(0.60,0.075)$ & 25.6 & 26.8 & 1245.4 & 11771.7 \\
$(0.60,0.09)$ & 25.6 & 26.8 & 1265.0 & 11706.1 \\
\hline
\end{tabular}

From the results in Table 1, we know that product defect rate has no effect on the wholesale and the buyback, but it has an impact on retailer's optimal order quantity and expected profit. Also it can be seen that if $\eta$ is a fixed value 0.6 then with the increase of $\alpha$, the optimal order quantity is increased for retailer but the expected profit of the retailer is reduced.

\section{CONCLUSIONS}

In this paper, we have investigated the fuzzy decision making problem when the real market demand couldn't have certain probability distribution or is not a crisp value and need to be estimated as fuzzy variable. And also we have considered the defect rate of the production to investigate buyback contract on the effectiveness of coordinating results. We have explored the optimal decision of supply chain system as well as suppliers and retailers in both defect free and defect. The model has been formulated based on credibility theory and also the optimal parameters of buyback contract are obtained through solving the presented model.
The numerical example shows that the defect rate has no effect on the wholesale and buyback price in the buyback contract, but for optimal ordering lots, retailer's expected profit and the profit of supply chain, the defect rate has different effects on them. The shortage of this study is that we have not taken into account the influence of vendor preferences on supply chain coordination, and also we have not do some comparison with buy back contract and other contracts in the coordination level. These are the issues to be addressed in the future.

\section{ACKNOWLEDGEMENTS}

This work was supported by Natural Science Foundation of Shandong Province (Grant No. ZR2010GL009) and 2011 International Cooperation Program of Shandong Provincial Education Department for Excellent Lecturers.

\section{REFERENCES}

[1] Gerard P. Cachon, "Supply chain coordination with contracts," in Graves S, De Kok T. The handbook of operations research and management science: supply chain management, Elsevier, Amsterdam: North Holland, 2003, pp.229-339.

[2] H. Emmons, S. Gilbert, "Return policies in pricing and inventory decisions for catalogue goods," Management Science, Vol.44, No.2, pp.276-283, 1998.

[3] V. Padmanabhan, I.P.L. Png, "Manufacturer's return policy and retail competition,” Marketing Science, Vol. 16, No.1, pp.81-94, 1997.

[4] H. Wang, W. Wang and K. Kobaccy, "Analysis and design of returns policies from a supplier's perspective," Journal of the Operational Research Society, Vol. 58, pp.391-401, 2007.

[5] S. Webster, Z. K. Weng, "Risk-free perishable item returns policy," Manufacturing and Service Operations Management, Vol.2, No.1, pp.100-106, 2000.

[6] Z. Yao, S. C H. Leung and K. K. Lai, "Analysis of the impact of price-sensitivity factors on the returns policy in coordinating supply chain," European Journal of Operational Research, Vol.187, pp.275282, 2008.

[7] Zhigang Zhao, Xiangyang Li and Xiuzhi Liu, "On response method for buyback contract with fuzzy random demand updating," Chinese Journa1 of Management Science, Vol. 15, No.3, pp.47-55, 2007. (in Chinese)

[8] Haijun Liu, Juhong Chen, "Supply chain fuzzy buyback contract based on context of unrepairable defect," Control and decision, Vol.25, No.8, pp.1155-1158, 1163, 2010. (in Chinese)

[9] Baoding Liu, Uncertain Theory: an Introduction to Its Axiomatic Foundations, Berlin, Germany: Springer-Verlag, 2004.

[10] Xiaobin Wang, Xiaohui Wang, "Supply chain coordination based on buy back contract with fuzzy demand," In Proceedings of the 8th International Conference on Machine Learning and Cybernetics, 2009, pp. 839-843.

[11] Shengju Sang, Qiang Zhang and Jianzhang Wu, "Coordination mechanism analysis for supply chain with fuzzy demand," Computer Integrated Manufacturing Systems, Vol. 16. No. 2, pp.357-379, 2010. (in Chinese)

[12] Junyan Wang, Wansheng Tang and Ruiqing Zhao, "Supply chain coordination by revenue-sharing contract with fuzzy demand," Journal of Intelligent and Fuzzy Systems, Vol.19, No.6, pp.409-420, 2008 . 